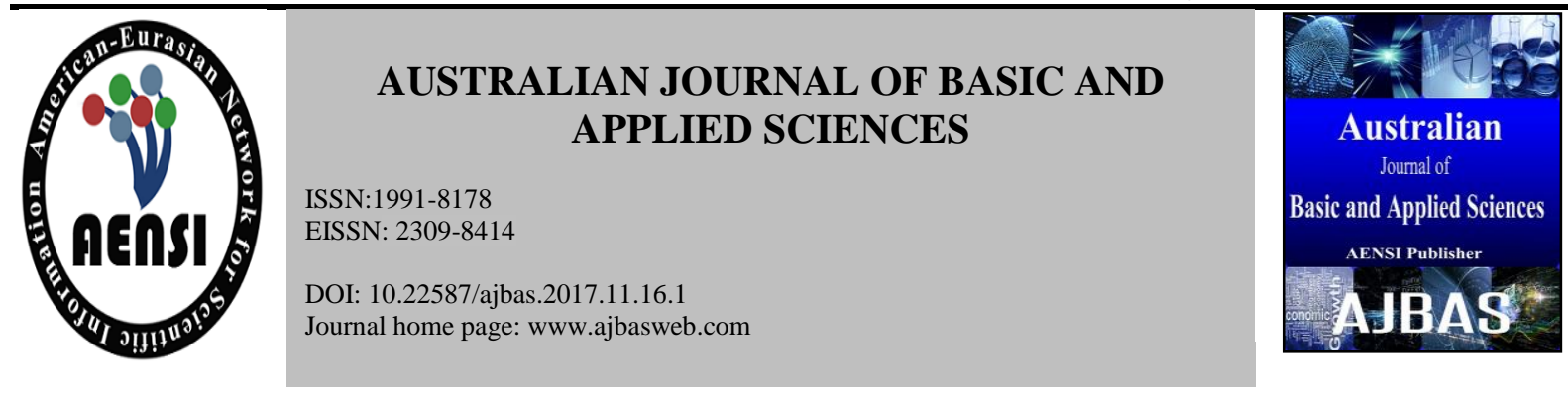

\title{
Two Suggested Approaches to Estimate Two Parameters Rayleigh Distribution
}

Firas A. Mohammed

Associated Professor, Department of Statistics College of Administration and Economic, University of Baghdad, Baghdad City, Iraq.

Address For Correspondence:

Firas A. Mohammed, Associated Professor, Department of Statistics College of Administration and Economic, University of Baghdad,

Baghdad City, Iraq.

E-mail: firasmohana@coadec.uobaghdad.edu.iq

\section{ART ICLE INFO}

\section{Article history:}

Received 12 October 2017

Accepted 22 December 2017

Available online 15 January 2018

\section{Keywords:}

Method of Moments, Modified

Maximum Likelihood, standardized

limits, six-sigma, hybrid approach.

\begin{abstract}
A B S T R A C T
Rayleigh distribution is a member of exponential family distributions that involved in life time testing machine components that studied mean time to failure. The Distribution has two parameters: shift parameter indicated by $(\alpha)$ and scale parameter $(\beta)$ described the behavior. Sometimes, the classical methods of estimating parameters of Rayleigh distribution suffered from weakness which results loss of significant information. Moreover, methods of artificial intelligence demonstrated a clear advantage of finding accurate estimation of the probability distribution curves in recent decades. The objective of this research is to introduce a promising estimation ad-hoc method in fast intelligent way that we call it (NEW-Sugg.) method based on nearest neighborhood technique in iterative manner. The (NEW-Sugg.) method was compared with other four estimation methods, three of them were classical methods: Maximum likelihood (ML) and Method of Moment (MOM).In order to complete the comparison, proposed maximum likelihood (Prop.ML) was performed. The optimality obtained from the comparison was investigated by using Mean Absolute Percentage (MAPE) criterion. Nine data sets of distinct empirical experiments were conducted with different values of parameters and different sample sizes. To conclude, MOM method got better results gradually as sample size grew for both $(\alpha, \beta)$.(MOM) \& (Prop.ML) be equivalent in $(\alpha)$ estimation, while (Prop.ML) got optimality in $(\beta)$ estimation. The major conclusion was the superiority of (NEW-Sugg.) method associated with all sample sizes for both parameters' estimations.
\end{abstract}

\section{INTRODUCTION}

The English physicist Lord Rayleigh (1880) wrote in this probability distribution firstly as mentioned Taha Anwar (2015). Rayleigh distribution is regarded as a member of exponential family distributions that involved in life time testing machine components that studied mean time to failure. Therefore, Rayleigh distribution is used because the life of the model theory reliability plays an important role in modeling the life of the random phenomenon as Andrew C. Mkolesia referred in his article (2016). Furthermore, Rayleigh distribution can be applied in organism's life expectancy especially in clinical trials in cancer studies and wind velocity.

Some recent contributions had been considered in this field such as: Smail Mahdi et al (2006) in comparison between Rayleigh with Logistic Distribution, Sanku Dey (2012) via introducing Bayesian estimation for inverse version of Rayleigh distribution which is improved by new weighted loss function by Huda A. Rasheed(2017). While Al-Kinani (2014) introduced emphasis comparison between Bayesian approach versus other Non-Bayesian one. The updating paper was formed by Fundi (2017) about estimating Rayleigh parameters under type II censoring data.

The importance of this paper is to introduce new contribution based on selecting combinations of classical estimations of two parameters of Rayleigh distribution, which are: maximum likelihood, method of moments,

Open Access Journal

Published BY AENSI Publication

(C) 2017 AENSI Publisher All rights reserved

This work is licensed under the Creative Commons Attribution International License (CC BY).

http://creativecommons.org/licenses/by/4.0/

c) (i) Open Access

To Cite This Article: Firas A. Mohammed., Two Suggested Approaches to Estimate Two Parameters Rayleigh Distribution. Aust. J. Basic \& Appl. Sci., 11(16):1-10, 2017 
least squares in addition to a proposed modified maximum likelihood. The NEW-Sugg. method regarded the previous estimation as input initial values and then begun to detect the best combination among them in nearest neighbor region (or interval) until satisfying the optimality.

\section{Rayleigh Distribution Characteristics:}

Rayleigh distribution is a member of exponential family distributions represented in life time such as: Gamma, Weibull, etc. It is a special case of Generalized Rayleigh Distribution described by A.A. Al-Naqeeb (2009).

Afify (2004) and Walck (2007) described Rayleigh distribution with two parameters $\alpha$ and $\beta$ has the probability density function below.

$f(x ; \alpha, \beta)=\frac{(x-\alpha)}{\beta^{2}} \exp \left\{-\frac{(x-\alpha)^{2}}{2 \beta^{2}}\right\} \quad ;$ where is: $x>\alpha \quad, \quad \beta>0$

The cumulative probability distribution function of equation (1) above is obtained by:

$F(x)=\operatorname{Pr}(X \leq x)=1-\exp \left\{-\frac{(x-\alpha)^{2}}{2 \beta^{2}}\right\}$

\section{Methods of Estimation:}

\section{Method of Moments:}

This method based on assumption that population moments of $\mathbf{r}^{\text {th }}$ order $\left(\mu_{r}\right)$ equals to sample moments of the same order $\left(m_{r}\right)$, and then solving the equations to find parameters' estimations, Li (2012) considered the procedure as follows:

$$
\begin{aligned}
& \mu_{r}=E\left(x^{r}\right) \\
& \mu_{1}=E(x) \\
& E(x)=\beta \sqrt{\frac{\pi}{2}}+\alpha \\
& \mu_{2}=E\left(x^{2}\right) \\
& E\left(x^{2}\right)=2 \beta^{2}+\sqrt{2 \pi} \alpha \beta+\alpha^{2}
\end{aligned}
$$

While the sample moment of order $\mathbf{r}$ is:

$$
\begin{gathered}
m_{r}=\frac{1}{n} \sum_{i=1}^{n}\left(x_{i}^{r}\right) \\
\therefore m_{1}=\frac{\sum_{i=1}^{n}\left(x_{i}\right)}{n}=\bar{x}
\end{gathered}
$$

By putting : $\mu_{1}=m_{1}$

$\hat{\beta} \sqrt{\frac{\pi}{2}}+\hat{\alpha}=\bar{x}$

$$
m_{2}=\frac{\sum_{i=1}^{n} x_{i}^{2}}{n}
$$

By putting : $\mu_{2}=m_{2}$ 
$2 \hat{\beta}^{2}+\sqrt{2 \pi} \hat{\alpha} \hat{\beta}+\hat{\alpha}^{2}=\frac{\sum_{i=1}^{n} x_{i}^{2}}{n}$

But; $\quad \frac{\sum_{i=1}^{n} x_{i}^{2}}{n}=\operatorname{Var}(x)+[E(x)]^{2}$

$S^{2}=\left(2-\frac{\pi}{2}\right) \hat{\beta}^{2}$

$\therefore S^{2}=\left(\frac{4-\pi}{2}\right) \hat{\beta}^{2}$

Where is : $\pi=\frac{22}{7}$

By solving the last equation above we get the moment estimation of $(\alpha) \&(\beta)$ are:

$$
\begin{aligned}
& \hat{\beta}_{\text {mom }}=\sqrt{\frac{2}{4-\pi}} S \\
& \hat{\alpha}_{\text {mom }}=\bar{x}-\sqrt{\frac{\pi}{2}} \hat{\beta}_{\text {mom }}
\end{aligned}
$$

S: the standard deviation of the data.

\section{Maximum Likelihood Method:}

The maximum likelihood method represents is one of the most significant method that maximize the likelihood for a r.v. follows Rayleigh distribution with location parameter $(\alpha)$ and scale parameter $(\beta)$. This method had a well discussion by Yahya (2013) and Ahmed (2013). We can get their estimates by following steps:

$$
\begin{array}{r}
L\left(x_{i} ; \alpha, \beta\right)=\pi_{i=1}^{n}\left[\frac{\left(x_{i}-\alpha\right)}{\beta^{2}} e^{-(x i-\alpha) / 2 \beta^{2}}\right] \\
L\left(x_{i} ; \alpha, \beta\right)=\frac{1}{\beta^{2 n}} \pi_{i=1}^{n}\left(x_{i}-\alpha\right) \exp \left\{\begin{array}{c}
\sum_{i=1}^{n}(x i-\alpha)^{2} \\
-\frac{i=1}{2 \beta^{2}}
\end{array}\right\}
\end{array}
$$

But, after taking logarithm:

$$
\ln L(x ; \alpha, \beta)=-2 n \ln (\beta)+\sum_{i=1}^{n} \ln \left(x_{i}-\alpha\right)-\frac{\sum_{i=1}^{n}\left(x_{i}-\alpha\right)^{2}}{2 \beta^{2}}
$$

with partial derivatives with respect to $(\alpha)$ and $(\beta)$ and equating them to zero respectively, we will get:

$$
\frac{\partial \ln L}{\partial \alpha}=0
$$


$-\sum_{i=1}^{n}\left(\frac{1}{x_{i}-\hat{\alpha}}\right)+\frac{\sum_{i=1}^{n} x_{i}-n \hat{\alpha}}{\hat{\beta}^{2}}=0$

Since, $\mathrm{x}_{\mathrm{i}}>\alpha \quad \rightarrow \quad \min \left(\mathrm{x}_{\mathrm{i}}\right) \geq \hat{\alpha}$ i.e.,

Hence, $\quad \alpha$ estimation will be merely the first ordered statistics of $\left(\mathrm{x}_{\mathrm{i}}\right)$ after sort them in ascending manner,

$\hat{\alpha}_{M L}=x_{(1)}$

Similarly, we can get ( $\beta$ ) estimation by:

$$
\begin{aligned}
& \frac{\partial \ln L}{\partial \beta}=0 \\
& -\frac{2 n}{\hat{\beta}}+\frac{\sum_{i=1}^{n}\left(x_{i}-\hat{\alpha}\right)^{2}}{\hat{\beta}^{2}}=0 \\
& \hat{\beta}_{M L}=\sqrt{\frac{\sum_{i=1}^{n}\left(x_{i}-\hat{\alpha}_{M L}\right)^{2}}{2 n}} \\
& \therefore \hat{\beta}_{M L}=\sqrt{\frac{\sum_{i=1}^{n}\left(x_{i}-x_{(1)}\right)^{2}}{2 n}}
\end{aligned}
$$

\section{Proposed Maximum Likelihood:}

We propose the following modification upon the classical Maximum Likelihood described above. We do this by using median value in two instead of mean of data.

i. Beginning the procedures with input data of $\left(\mathrm{x}_{\mathrm{i}}\right)$.

ii. Calculate $\mu_{\mathrm{e}}$ as the median of $\left(\mathrm{x}_{\mathrm{i}}\right)$.

iii. Find the Mean Absolute of Deviation of $\mu_{\mathrm{e}}$ which is given by:

$S_{A}=\frac{1}{n} \sum_{i=1}^{n}\left|x_{i}-\mu_{e}\right|$

iv. Initializing two parameters with values:

$\beta_{0}=S_{A} \cdot \sqrt{\frac{2}{4-\pi}}$

$\alpha_{0}=\bar{x}-\beta_{0} \sqrt{\frac{\pi}{2}}$

v. Enter iterative procedure with the following:

$\hat{\beta}_{i .}=\sqrt{\frac{\sum_{i=1}^{n}\left(x_{i}-\hat{\alpha}_{i-1 .}\right)^{2}}{2 n}}$

$\hat{\alpha}_{i .}=\bar{x}-\hat{\beta}_{i-1} \sqrt{\frac{\pi}{2}}$

vi. Construct the stopping rule (TD) interms of total deviation as below. 
$T D=\frac{\left|\hat{\alpha}_{i}-\hat{\alpha}_{i-1}\right|+\left|\hat{\beta}_{i}-\hat{\beta}_{i-1}\right|}{2}$

vii. Stop if $T D \leq 0.0001$ and obtain: $\left(\hat{\alpha}_{i .}, \hat{\beta}_{i}\right)$ at final.

\section{The NEW-Sugg. suggested Method:}

In seeking a new promising hybrid approach by obtaining the estimation of parameters by indicating the nearest neighbor strategy in iterative manner in six-sigma pattern.

The essential thing is to achieve maxima (or minima) in wide scope of point estimations comes originally from initial values and their neighbors as selected samples and then resampling alternative points iteratively.

$1^{\text {st }}$ Stage: Initialize procedure with values of the couple parameters $(\alpha, \beta)$ by choosing the classical estimations that got in advance: Ml \& Mom in addition to the Prop.ML as "seeds".

$2^{\text {nd }}$ Stage: Select the best combination estimation $\left(\alpha_{0}, \beta_{0}\right)$ according to (MAPE) criterion.

$3^{\text {rd }}$ Stage: Construct an area performed by points (say; $\left.\alpha_{0 \mathrm{i}}, \beta_{0 \mathrm{i}}\right) ; \mathrm{i}=1,2,3, \ldots, 200$ that belonging to the window $\left\{\alpha_{0 \mathrm{i}} \in\left(\alpha_{0 \mathrm{i}} \mathrm{i} \operatorname{Sig}(\alpha), \beta_{0 \mathrm{j}} \in\left(\beta_{0 \mathrm{i}} \pm \operatorname{Sig}(\beta)\right\}\right.\right.$, where is:

$\operatorname{Sig}(\varphi)=6 * \sqrt{\frac{\sum_{j=1}^{r}\left(\varphi_{0 i j}-\varphi_{0 i}\right)^{2}}{r}}$

Where is $(\varphi)$ represents $(\boldsymbol{\alpha})$ or $(\boldsymbol{\beta})$.

$r$ : represents number of replicates.

(i): is one of the four methods.

$4^{\text {th }}$ Stage: Make comparisons among the selected best method $\left(\alpha_{0}, \beta_{\mathrm{o}}\right)$ got in step $(2)$ against the $\left(\alpha_{0 \mathrm{i}}, \beta_{0 \mathrm{i}}\right)$ to get the required, regarded as candidate estimations in successive comparisons among them against $\left(\alpha_{0}, \beta_{\mathrm{o}}\right)$ via (MAPE) until getting settle down in convergence stability.

$5^{\text {th }}$ Stage: Going on NEW-Sugg. estimations consequently in alternative approach until obtain $\left(\hat{\alpha}_{\text {sugg. }}, \hat{\beta}_{\text {sugg. }}\right)$ finally.

\section{Comparison Criteria:}

Suppose $(\varphi)$ be one of the two parameters of Rayleigh distribution $(\lambda)$ or $(\theta)$. Let $(\mathbf{r})$ be the replications.

Mean Absolute Percentage Error (MAPE)

$M A P E=\frac{1}{r e p} \sum_{i=1}^{r e p}\left|\frac{\varphi_{i}-\hat{\varphi}_{i}}{\varphi_{i}}\right|$

Rashid et al (2011) used Total Deviations (TD)

$T D=\frac{1}{r e p} \sum_{i=1}^{r e p}\left(\left|\frac{\hat{\alpha}_{i}-\alpha_{i}}{\alpha_{i}}\right|+\left|\frac{\hat{\beta}_{i}-\beta_{i}}{\beta_{i}}\right|\right)$

\section{Empirical Simulations:}

The model represents the system itself, while the simulation represents the operation of the system over time. Computer simulations have become a useful part of mathematical modeling of many natural systems in sciences .So, the simulation is a type of sampling techniques as had been referred by Taha (2015).

Simulations for both parameters $(\alpha$ and $\beta$ ) had been done as follows:

$\alpha=(0.50,0.75,1.0)$

$\beta=(1,2,3.5)$

$\mathrm{n}=(10,25,50,200)$

The numerical results in the following tables: $(1,2,3,4,5,6$ and 7$)$ as below. 


\section{Discussion and Conclusion:}

On the light of experimental results got above, both parameters $(\alpha, \beta)$ of Rayleigh distribution are estimated using five methods that compared by (MAPE). We can list the following conclusions:

a) In general, it can be noticed that (NEW-SUGG.) suggested method the best than the other methods for both parameters with respect to (MAPE) criterion and for all sample sizes.

b) (MOM) method got better gradually as sample size grew for $\operatorname{MAPE}(\alpha)$ compared with (ML) and (Prop.ML), while it got closer to (Prop.ML) as sample sizes $(10,25)$, i.e. both of (MOM) \& (Prop.ML) be equivalent and better than the other two methods (ML). On other hand, (MOM)and (Prop.ML) got more closeness at the sample sizes $(50,200)$.

c) (Prop.ML) is the second best method in estimation after (NEW-SUGG.) for MAPE( $\beta$ ) in Table (3) compared with the rest four methods with all sample sizes.

d) (MOM) method got better results gradually as sample size grew for MAPE $(\beta)$ in Table (4) compared with (ML) while it got closer to (Prop.ML) as sample sizes (200).

e) The Replicates in Table (6) showed that (MOM) had the major contribution that got the best sharing in (NEW-Sugg.) method optimization.

f) The Replicates in Table (7) reflect the competition in global superiority among the five methods that represented as: (MOM and Prop.ML) when ordered in descending respectively. While the (NEW-SUGG.) Method beats them all.

Table 1: Estimated $(\alpha)$

\begin{tabular}{|c|c|c|c|c|c|c|}
\hline \multirow[b]{2}{*}{$\mathrm{n}$} & \multirow[b]{2}{*}{$\alpha$} & \multirow[b]{2}{*}{$\beta$} & \multicolumn{4}{|c|}{ Methods of Estimation } \\
\hline & & & MLE & MOM & Prop.ML & NEW-SUGG. \\
\hline \multirow{9}{*}{10} & 1 & 0.5 & 1.197849 & 1.017038 & 1.049020 & 1.001720 \\
\hline & & 0.75 & 1.300470 & 1.028457 & 1.075651 & 1.004197 \\
\hline & & 1.0 & 1.404631 & 1.044674 & 1.107472 & 1.005660 \\
\hline & 2 & 0.5 & 2.203064 & 2.025092 & 2.056488 & 2.003233 \\
\hline & & 0.75 & 2.302654 & 2.028737 & 2.075870 & 2.003785 \\
\hline & & 1.0 & 2.393647 & 2.037441 & 2.100209 & 2.004668 \\
\hline & 3.5 & 0.5 & 3.704836 & 3.523060 & 3.554418 & 3.502553 \\
\hline & & 0.75 & 3.803400 & 3.541135 & 3.587641 & 3.502497 \\
\hline & & 1.0 & 3.887730 & 3.518989 & 3.582646 & 3.503420 \\
\hline \multirow{9}{*}{25} & 1 & 0.5 & 1.123654 & 1.005349 & 1.018341 & 1.001293 \\
\hline & & 0.75 & 1.188789 & 1.014976 & 1.034021 & 1.001956 \\
\hline & & 1.0 & 1.251342 & 1.023856 & 1.049107 & 1.002061 \\
\hline & 2 & 0.5 & 2.123805 & 2.004390 & 2.017383 & 2.000744 \\
\hline & & 0.75 & 2.189956 & 2.017507 & 2.036540 & 2.001239 \\
\hline & & 1.0 & 2.250384 & 2.013691 & 2.039182 & 2.003011 \\
\hline & 3.5 & 0.5 & 3.625259 & 3.506241 & 3.519171 & 3.501171 \\
\hline & & 0.75 & 3.689612 & 3.506058 & 3.525326 & 3.501168 \\
\hline & & 1.0 & 3.755704 & 3.511482 & 3.536859 & 3.501957 \\
\hline \multirow{9}{*}{50} & 1 & 0.5 & 1.088491 & 1.003633 & 1.010283 & 1.000790 \\
\hline & & 0.75 & 1.130233 & 1.004031 & 1.013838 & 1.001594 \\
\hline & & 1.0 & 1.176157 & 1.008075 & 1.020961 & 1.002179 \\
\hline & 2 & 0.5 & 2.089131 & 2.003728 & 2.010399 & 2.001151 \\
\hline & & 0.75 & 2.132597 & 2.007699 & 2.017480 & 2.000812 \\
\hline & & 1.0 & 2.180145 & 2.011511 & 2.024385 & 2.001568 \\
\hline & 3.5 & 0.5 & 3.589863 & 3.505828 & 3.512485 & 3.500435 \\
\hline & & 0.75 & 3.631736 & 3.503279 & 3.513070 & 3.501379 \\
\hline & & 1.0 & 3.677461 & 3.510005 & 3.522902 & 3.501785 \\
\hline \multirow{9}{*}{200} & 1 & 0.5 & 1.045197 & 1.001855 & 1.003821 & 1.000685 \\
\hline & & 0.75 & 1.065182 & 0.998670 & 1.001425 & 1.000802 \\
\hline & & 1.0 & 1.091314 & 1.005998 & 1.009526 & 1.001591 \\
\hline & 2 & 0.5 & 2.043725 & 2.000494 & 2.002462 & 2.000676 \\
\hline & & 0.75 & 2.065665 & 1.998602 & 2.001349 & 2.000724 \\
\hline & & 1.0 & 2.088025 & 1.999082 & 2.002620 & 2.001117 \\
\hline & 3.5 & 0.5 & 3.544377 & 3.499869 & 3.501839 & 3.500693 \\
\hline & & 0.75 & 3.566449 & 3.500818 & 3.503569 & 3.500690 \\
\hline & & 1.0 & 3.587769 & 3.500062 & 3.503592 & 3.500871 \\
\hline
\end{tabular}


Table 2: Estimated ( $\beta$ )

\begin{tabular}{|c|c|c|c|c|c|c|}
\hline & & & Methods o & & & \\
\hline $\mathrm{n}$ & $\alpha$ & $\beta$ & MLE & MOM & Prop.ML & NEW-SUGG. \\
\hline \multirow{9}{*}{10} & 1 & 0.5 & 0.377184 & 0.491037 & 0.465588 & 0.498817 \\
\hline & & 0.75 & 0.556336 & 0.727566 & 0.689979 & 0.747367 \\
\hline & & 1.0 & 0.743547 & 0.970168 & 0.920131 & 0.997600 \\
\hline & 2 & 0.5 & 0.369864 & 0.481962 & 0.456980 & 0.498321 \\
\hline & & 0.75 & 0.554138 & 0.726598 & 0.689061 & 0.748137 \\
\hline & & 1.0 & 0.745146 & 0.969738 & 0.919724 & 0.997961 \\
\hline & 3.5 & 0.5 & 0.367003 & 0.481348 & 0.456397 & 0.498771 \\
\hline & & 0.75 & 0.551552 & 0.716872 & 0.679834 & 0.748351 \\
\hline & & 1.0 & 0.751685 & 0.983523 & 0.932801 & 0.997415 \\
\hline \multirow{9}{*}{25} & 1 & 0.5 & 0.423268 & 0.497285 & 0.486988 & 0.499720 \\
\hline & & 0.75 & 0.627664 & 0.736369 & 0.721241 & 0.749202 \\
\hline & & 1.0 & 0.839030 & 0.981357 & 0.961279 & 0.998681 \\
\hline & 2 & 0.5 & 0.422662 & 0.497275 & 0.486977 & 0.499751 \\
\hline & & 0.75 & 0.627978 & 0.735842 & 0.720725 & 0.749495 \\
\hline & & 1.0 & 0.842942 & 0.990888 & 0.970618 & 0.998530 \\
\hline & 3.5 & 0.5 & 0.420525 & 0.494833 & 0.484584 & 0.499407 \\
\hline & & 0.75 & 0.630584 & 0.745195 & 0.729890 & 0.749358 \\
\hline & & 1.0 & 0.833948 & 0.986332 & 0.966153 & 0.998423 \\
\hline \multirow{9}{*}{50} & 1 & 0.5 & 0.443294 & 0.496271 & 0.491033 & 0.499707 \\
\hline & & 0.75 & 0.668108 & 0.746990 & 0.739233 & 0.749035 \\
\hline & & 1.0 & 0.886128 & 0.991177 & 0.980964 & 0.999084 \\
\hline & 2 & 0.5 & 0.444528 & 0.497860 & 0.492607 & 0.499588 \\
\hline & & 0.75 & 0.666810 & 0.744834 & 0.737098 & 0.749491 \\
\hline & & 1.0 & 0.885065 & 0.990387 & 0.980183 & 0.999337 \\
\hline & 3.5 & 0.5 & 0.444220 & 0.496772 & 0.491529 & 0.499794 \\
\hline & & 0.75 & 0.665352 & 0.745593 & 0.737849 & 0.748885 \\
\hline & & 1.0 & 0.887361 & 0.992084 & 0.981862 & 0.999223 \\
\hline \multirow{9}{*}{200} & 1 & 0.5 & 0.471719 & 0.498812 & 0.497312 & 0.499593 \\
\hline & & 0.75 & 0.709350 & 0.750894 & 0.748764 & 0.749499 \\
\hline & & 1.0 & 0.943962 & 0.997314 & 0.994567 & 0.999086 \\
\hline & 2 & 0.5 & 0.472607 & 0.499617 & 0.498116 & 0.499618 \\
\hline & & 0.75 & 0.706727 & 0.748614 & 0.746490 & 0.749536 \\
\hline & & 1.0 & 0.945170 & 1.000723 & 0.997968 & 0.999326 \\
\hline & 3.5 & 0.5 & 0.472523 & 0.500317 & 0.498814 & 0.499608 \\
\hline & & 0.75 & 0.708040 & 0.749060 & 0.746934 & 0.749602 \\
\hline & & 1.0 & 0.942745 & 0.997532 & 0.994785 & 0.999266 \\
\hline
\end{tabular}

Table 3: $\operatorname{MAPE}(\alpha)$

\begin{tabular}{|c|c|c|c|c|c|c|}
\hline \multirow[b]{2}{*}{$\mathrm{n}$} & \multirow[b]{2}{*}{$\alpha$} & \multirow[b]{2}{*}{$\beta$} & \multicolumn{4}{|c|}{ Methods of Estimation } \\
\hline & & & MLE & MOM & Prop.ML & NEW-SUGG. \\
\hline \multirow{9}{*}{10} & 1 & 0.5 & 0.197849 & 0.119418 & 0.121266 & 0.002376 \\
\hline & & 0.75 & 0.300470 & 0.173852 & 0.175242 & 0.005128 \\
\hline & & 1.0 & 0.404631 & 0.223424 & 0.229109 & 0.006904 \\
\hline & 2 & 0.5 & 0.101532 & 0.056979 & 0.058440 & 0.001967 \\
\hline & & 0.75 & 0.151327 & 0.084946 & 0.086552 & 0.002388 \\
\hline & & 1.0 & 0.196823 & 0.111563 & 0.114022 & 0.003006 \\
\hline & 3.5 & 0.5 & 0.058524 & 0.032776 & 0.033202 & 0.000930 \\
\hline & & 0.75 & 0.086686 & 0.048437 & 0.050153 & 0.001028 \\
\hline & & 1.0 & 0.110780 & 0.066383 & 0.066369 & 0.001332 \\
\hline \multirow{9}{*}{25} & 1 & 0.5 & 0.123654 & 0.068344 & 0.068852 & 1.001293 \\
\hline & & 0.75 & 0.188789 & 0.104860 & 0.105594 & 0.002595 \\
\hline & & 1.0 & 0.251342 & 0.142847 & 0.145363 & 0.002895 \\
\hline & 2 & 0.5 & 0.061903 & 0.036243 & 0.036353 & 0.000579 \\
\hline & & 0.75 & 0.094978 & 0.052516 & 0.053464 & 0.000956 \\
\hline & & 1.0 & 0.125192 & 0.070742 & 0.071099 & 0.001931 \\
\hline & 3.5 & 0.5 & 0.035788 & 0.020672 & 0.020911 & 0.000463 \\
\hline & & 0.75 & 0.054175 & 0.030727 & 0.030824 & 0.000517 \\
\hline & & 1.0 & 0.073058 & 0.041470 & 0.041616 & 0.000811 \\
\hline \multirow{9}{*}{50} & 1 & 0.5 & 0.088491 & 0.049824 & 0.050138 & 0.001097 \\
\hline & & 0.75 & 0.130233 & 0.075687 & 0.076047 & 0.002037 \\
\hline & & 1.0 & 0.176157 & 0.101285 & 0.101745 & 0.002778 \\
\hline & 2 & 0.5 & 0.044565 & 0.025724 & 0.025829 & 0.000719 \\
\hline & & 0.75 & 0.066298 & 0.037782 & 0.038071 & 0.000632 \\
\hline & & 1.0 & 0.090072 & 0.048317 & 0.048659 & 0.001069 \\
\hline & 3.5 & 0.5 & 0.025675 & 0.013890 & 0.013995 & 0.000204 \\
\hline & & 0.75 & 0.037639 & 0.021794 & 0.021851 & 0.000520 \\
\hline & & 1.0 & 0.050703 & 0.028018 & 0.028107 & 0.000684 \\
\hline \multirow[t]{2}{*}{200} & 1 & 0.5 & 0.045197 & 0.025420 & 0.025593 & 0.000834 \\
\hline & & 0.75 & 0.065182 & 0.036612 & 0.036633 & 0.000997 \\
\hline
\end{tabular}




\begin{tabular}{|l|l|l|l|l|l|l|}
\hline & & 1.0 & 0.091314 & 0.049340 & 0.049621 & 0.001869 \\
\cline { 2 - 6 } & 2 & 0.5 & 0.021863 & 0.012815 & 0.012818 & 0.000412 \\
\cline { 2 - 6 } & 0.75 & 0.032833 & 0.018721 & 0.018647 & 0.000477 \\
\cline { 2 - 6 } & 3.5 & 0.0 & 0.044012 & 0.025148 & 0.025143 & 0.000716 \\
\cline { 2 - 6 } & & 0.75 & 0.018798 & 0.007445 & 0.007454 & 0.000248 \\
\cline { 2 - 6 } & 1.0 & 0.025077 & 0.013952 & 0.010529 & 0.000262 \\
\hline
\end{tabular}

Table 4: $\operatorname{MAPE}(\beta)$

\begin{tabular}{|c|c|c|c|c|c|c|}
\hline & & & Methods o & & & \\
\hline $\mathrm{n}$ & $\alpha$ & $\beta$ & MLE & MOM & Prop.ML & NEW-SUGG. \\
\hline \multirow{9}{*}{10} & 1 & 0.5 & 0.260908 & 0.192968 & 0.192520 & 0.003506 \\
\hline & & 0.75 & 0.271121 & 0.198755 & 0.200265 & 0.004868 \\
\hline & & 1.0 & 0.266304 & 0.189710 & 0.191589 & 0.003739 \\
\hline & 2 & 0.5 & 0.277043 & 0.203761 & 0.207237 & 0.004801 \\
\hline & & 0.75 & 0.273563 & 0.200675 & 0.201414 & 0.003883 \\
\hline & & 1.0 & 0.269265 & 0.190792 & 0.192747 & 0.003294 \\
\hline & 3.5 & 0.5 & 0.277166 & 0.196948 & 0.200039 & 0.003928 \\
\hline & & 0.75 & 0.275967 & 0.194247 & 0.198483 & 0.003474 \\
\hline & & 1.0 & 0.265556 & 0.200740 & 0.200986 & 0.003930 \\
\hline \multirow{9}{*}{25} & 1 & 0.5 & 0.163592 & 0.119489 & 0.119500 & 0.001416 \\
\hline & & 0.75 & 0.173834 & 0.120406 & 0.121578 & 0.001867 \\
\hline & & 1.0 & 0.170010 & 0.123030 & 0.124163 & 0.002152 \\
\hline & 2 & 0.5 & 0.165025 & 0.121839 & 0.121616 & 0.001345 \\
\hline & & 0.75 & 0.172264 & 0.119182 & 0.120795 & 0.001483 \\
\hline & & 1.0 & 0.165845 & 0.120618 & 0.121123 & 0.002358 \\
\hline & 3.5 & 0.5 & 0.165848 & 0.119060 & 0.120117 & 0.002042 \\
\hline & & 0.75 & 0.168212 & 0.121224 & 0.121022 & 0.001785 \\
\hline & & 1.0 & 0.173143 & 0.122012 & 0.122530 & 0.002408 \\
\hline \multirow{9}{*}{50} & 1 & 0.5 & 0.119837 & 0.083771 & 0.084348 & 0.001164 \\
\hline & & 0.75 & 0.115321 & 0.085811 & 0.085804 & 0.001898 \\
\hline & & 1.0 & 0.121528 & 0.086457 & 0.086793 & 0.001509 \\
\hline & 2 & 0.5 & 0.117872 & 0.083751 & 0.084027 & 0.001343 \\
\hline & & 0.75 & 0.119692 & 0.086217 & 0.086404 & 0.001320 \\
\hline & & 1.0 & 0.121137 & 0.080039 & 0.080866 & 0.001238 \\
\hline & 3.5 & 0.5 & 0.118180 & 0.080749 & 0.081172 & 0.001044 \\
\hline & & 0.75 & 0.119398 & 0.087160 & 0.087158 & 0.002129 \\
\hline & & 1.0 & 0.118580 & 0.085152 & 0.085395 & 0.001442 \\
\hline \multirow{9}{*}{200} & 1 & 0.5 & 0.060076 & 0.041494 & 0.041591 & 0.001142 \\
\hline & & 0.75 & 0.057827 & 0.040752 & 0.040785 & 0.000970 \\
\hline & & 1.0 & 0.060138 & 0.041744 & 0.041801 & 0.001209 \\
\hline & 2 & 0.5 & 0.059552 & 0.042715 & 0.042717 & 0.001073 \\
\hline & & 0.75 & 0.061148 & 0.042057 & 0.042017 & 0.000965 \\
\hline & & 1.0 & 0.058743 & 0.041810 & 0.041819 & 0.000968 \\
\hline & 3.5 & 0.5 & 0.058795 & 0.043542 & 0.043489 & 0.001110 \\
\hline & & 0.75 & 0.059638 & 0.042113 & 0.042162 & 0.000840 \\
\hline & & 1.0 & 0.061578 & 0.042900 & 0.042960 & 0.001052 \\
\hline
\end{tabular}

Table 5: $\operatorname{TD}(\alpha, \beta)$

\begin{tabular}{|c|c|c|c|c|c|c|}
\hline & & & Methods o & & & \\
\hline $\mathrm{n}$ & $\alpha$ & $\beta$ & MLE & MOM & Prop.ML & NEW-SUGG. \\
\hline \multirow{9}{*}{10} & 1 & 0.5 & 0.458758 & 0.312386 & 0.313786 & 0.005882 \\
\hline & & 0.75 & 0.571592 & 0.372608 & 0.375508 & 0.009996 \\
\hline & & 1.0 & 0.670936 & 0.413134 & 0.420698 & 0.010642 \\
\hline & 2 & 0.5 & 0.378576 & 0.260740 & 0.265678 & 0.006768 \\
\hline & & 0.75 & 0.424890 & 0.285622 & 0.287966 & 0.006272 \\
\hline & & 1.0 & 0.466088 & 0.302356 & 0.306770 & 0.006300 \\
\hline & 3.5 & 0.5 & 0.335690 & 0.229724 & 0.233242 & 0.004858 \\
\hline & & 0.75 & 0.362652 & 0.242684 & 0.248636 & 0.004502 \\
\hline & & 1.0 & 0.376336 & 0.267122 & 0.267354 & 0.005262 \\
\hline \multirow{9}{*}{25} & 1 & 0.5 & 0.287246 & 0.187832 & 0.188352 & 0.003152 \\
\hline & & 0.75 & 0.362622 & 0.225266 & 0.227172 & 0.004462 \\
\hline & & 1.0 & 0.421352 & 0.265878 & 0.269526 & 0.005048 \\
\hline & 2 & 0.5 & 0.226928 & 0.158082 & 0.157968 & 0.001924 \\
\hline & & 0.75 & 0.267242 & 0.171698 & 0.174258 & 0.002438 \\
\hline & & 1.0 & 0.291036 & 0.191360 & 0.192222 & 0.004288 \\
\hline & 3.5 & 0.5 & 0.201636 & 0.139732 & 0.141028 & 0.002504 \\
\hline & & 0.75 & 0.222388 & 0.151952 & 0.151846 & 0.002302 \\
\hline & & 1.0 & 0.246202 & 0.163482 & 0.164146 & 0.003220 \\
\hline \multirow[t]{3}{*}{50} & 1 & 0.5 & 0.208328 & 0.133594 & 0.134486 & 0.002260 \\
\hline & & 0.75 & 0.245554 & 0.161498 & 0.161850 & 0.003934 \\
\hline & & 1.0 & 0.297686 & 0.187742 & 0.188538 & 0.004286 \\
\hline
\end{tabular}


Firas A. Mohammed, 2017 Australian Journal of Basic and Applied Sciences, 11(16) December 2017, Pages: 1-10

\begin{tabular}{|c|c|c|c|c|c|c|}
\hline & 2 & 0.5 & 0.162438 & 0.109474 & 0.109856 & 0.002062 \\
\hline & 3.5 & 0.5 & 0.143856 & 0.094640 & 0.095168 & 0.001248 \\
\hline & & 1.0 & 0.169284 & 0.113170 & 0.113502 & 0.002126 \\
\hline \multirow{7}{*}{200} & 1 & 0.5 & 0.105272 & 0.066914 & 0.067184 & 0.001976 \\
\hline & & 0.75 & 0.123010 & 0.077364 & 0.077418 & 0.001968 \\
\hline & & 0.75 & 0.093980 & 0.060778 & 0.060664 & 0.001442 \\
\hline & & 1.0 & 0.102756 & 0.066958 & 0.066962 & 0.001684 \\
\hline & 3.5 & 0.5 & 0.071474 & 0.050986 & 0.050942 & 0.001358 \\
\hline & & 0.75 & 0.078624 & 0.052626 & 0.052690 & 0.001102 \\
\hline & & 1.0 & 0.086654 & 0.056852 & 0.056870 & 0.001392 \\
\hline
\end{tabular}

Table 6: Replicates of the number of Best Method Occurred

\begin{tabular}{|c|c|c|c|c|c|}
\hline \multirow[b]{2}{*}{$\mathrm{n}$} & \multirow[b]{2}{*}{$\alpha$} & \multirow[b]{2}{*}{$\beta$} & \multicolumn{3}{|c|}{ Methods of Estimation } \\
\hline & & & MLE & MOM & Prop.ML \\
\hline \multirow{9}{*}{10} & 1 & 0.5 & 177 & 156 & 233 \\
\hline & & 0.75 & 155 & 145 & 253 \\
\hline & & 1.0 & 138 & 175 & 262 \\
\hline & 2 & 0.5 & 163 & 145 & 207 \\
\hline & & 0.75 & 151 & 146 & 245 \\
\hline & & 1.0 & 146 & 150 & 249 \\
\hline & 3.5 & 0.5 & 170 & 145 & 209 \\
\hline & & 0.75 & 164 & 165 & 214 \\
\hline & & 1.0 & 198 & 161 & 211 \\
\hline \multirow{9}{*}{25} & 1 & 0.5 & 204 & 125 & 264 \\
\hline & & 0.75 & 168 & 120 & 275 \\
\hline & & 1.0 & 167 & 136 & 285 \\
\hline & 2 & 0.5 & 227 & 95 & 249 \\
\hline & & 0.75 & 196 & 135 & 229 \\
\hline & & 1.0 & 194 & 144 & 256 \\
\hline & 3.5 & 0.5 & 217 & 120 & 231 \\
\hline & & 0.75 & 220 & 117 & 248 \\
\hline & & 1.0 & 186 & 119 & 264 \\
\hline \multirow{9}{*}{50} & 1 & 0.5 & 218 & 114 & 263 \\
\hline & & 0.75 & 201 & 85 & 299 \\
\hline & & 1.0 & 183 & 96 & 311 \\
\hline & 2 & 0.5 & 216 & 90 & 264 \\
\hline & & 0.75 & 207 & 83 & 278 \\
\hline & & 1.0 & 192 & 108 & 267 \\
\hline & 3.5 & $\begin{array}{l}0.5 \\
\end{array}$ & 228 & 107 & 255 \\
\hline & & 0.75 & 239 & 90 & 254 \\
\hline & & 1.0 & 237 & 87 & 243 \\
\hline \multirow{9}{*}{200} & 1 & 0.5 & 227 & 61 & 293 \\
\hline & & 0.75 & 234 & 74 & 302 \\
\hline & & 1.0 & 193 & 83 & 308 \\
\hline & 2 & 0.5 & 259 & 61 & 275 \\
\hline & & 0.75 & 244 & 54 & 306 \\
\hline & & 1.0 & 259 & 77 & 279 \\
\hline & 3.5 & 0.5 & 273 & 59 & 273 \\
\hline & & 0.75 & 244 & 67 & 278 \\
\hline & & 1.0 & 262 & 69 & 275 \\
\hline
\end{tabular}

Table 7: Replicates of the number of Best Method Occurred

\begin{tabular}{|c|c|c|c|c|c|c|}
\hline \multirow[b]{2}{*}{$\mathrm{n}$} & \multirow[b]{2}{*}{$\alpha$} & \multirow[b]{2}{*}{$\beta$} & \multicolumn{4}{|c|}{ Methods of Estimation } \\
\hline & & & MLE & MOM & Prop.ML & NEW-SUGG. \\
\hline 10 & 1 & 0.5 & 0 & 0 & 0 & 1000 \\
\hline 10 & & 0.75 & 0 & 0 & 0 & 1000 \\
\hline 10 & & 1.0 & 0 & 0 & 0 & 1000 \\
\hline 10 & 2 & 0.5 & 0 & 0 & 0 & 1000 \\
\hline 10 & & 0.75 & 0 & 0 & 1 & 1000 \\
\hline 10 & & 1.0 & 0 & 0 & 0 & 1000 \\
\hline 10 & 3.5 & 0.5 & 0 & 1 & 1 & 1000 \\
\hline 10 & & 0.75 & 0 & 0 & 0 & 1000 \\
\hline 10 & & 1.0 & 0 & 0 & 0 & 1000 \\
\hline 25 & 1 & 0.5 & 0 & 1 & 0 & 1000 \\
\hline 25 & & 0.75 & 0 & 1 & 0 & 1000 \\
\hline 25 & & 1.0 & 0 & 0 & 0 & 1000 \\
\hline 25 & 2 & 0.5 & 0 & 0 & 0 & 1000 \\
\hline
\end{tabular}




\begin{tabular}{|c|c|c|c|c|c|c|}
\hline 25 & & 0.75 & 0 & 1 & 0 & 1000 \\
\hline 25 & & 1.0 & 0 & 0 & 0 & 1000 \\
\hline 25 & 3.5 & 0.5 & 0 & 0 & 0 & 1000 \\
\hline 25 & & 0.75 & 0 & 0 & 2 & 1000 \\
\hline 25 & & 1.0 & 0 & 0 & 0 & 1000 \\
\hline 50 & 1 & 0.5 & 0 & 0 & 0 & 1000 \\
\hline 50 & & 0.75 & 0 & 0 & 0 & 1000 \\
\hline 50 & & 1.0 & 0 & 0 & 0 & 1000 \\
\hline 50 & 2 & 0.5 & 0 & 0 & 0 & 1000 \\
\hline 50 & & 0.75 & 0 & 1 & 0 & 1000 \\
\hline 50 & & 1.0 & 0 & 0 & 0 & 1000 \\
\hline 50 & 3.5 & 0.5 & 0 & 0 & 0 & 1000 \\
\hline 50 & & 0.75 & 0 & 0 & 1 & 1000 \\
\hline 50 & & 1.0 & 0 & 0 & 0 & 1000 \\
\hline 200 & 1 & 0.5 & 0 & 1 & 0 & 1000 \\
\hline 200 & & 0.75 & 0 & 0 & 0 & 1000 \\
\hline 200 & & 1.0 & 0 & 0 & 0 & 1000 \\
\hline 200 & 2 & 0.5 & 0 & 0 & 0 & 1000 \\
\hline 200 & & 0.75 & 0 & 0 & 0 & 1000 \\
\hline 200 & & 1.0 & 0 & 0 & 0 & 1000 \\
\hline 200 & 3.5 & 0.5 & 0 & 0 & 0 & 1000 \\
\hline 200 & & 0.75 & 0 & 0 & 0 & 1000 \\
\hline 200 & & 1.0 & 0 & 0 & 0 & 1000 \\
\hline
\end{tabular}

\section{REFERENCES}

AL-Naqeeb, A.A. and A.M. Hamed, 2009. "Estimation of the two parameters for Generalized Rayleigh Distribution Function using simulation Technique", Ibn Al- Haitham J. for Pure \& Appl. SCI., 22.

Afify, E.E., 2004. "Comparison of Estimators of Parameters for the Rayleigh Distribution", Faculty of Eng. Shibeen El KomMenoufia University.

Ahmed, A., S.P. Ahmed and J.A. Reshi, 2013. "Bayesian Analysis of Rayleigh Distribution",International Journal of Scientific and Research Publications, 3(10): 1-9.

Al - Kanani, Iden Hassan and Jasim, Shaima Abbas, 2014. "Non-Bayesian and Bayesian Estimation for Generalized Rayleigh Distribution", International Journal of Modern Mathematical Sciences, 10(2): 103-115.

Dey, Sanku, 2012. "Bayesian Estimation of the Parameter and Reliability Function of an Inverse Rayleigh Distribution", Malaysian Journal of Mathematical Sciences, 6(1): 113-124.

Fundi, Murithi Daniel, Njenga, Edward Gachangi and Keitany, Kemboi George, 2017. "Estimation of Parameters of the Two-Parameter RayleighDistribution Based on Progressive Type-II Censoring Using Maximum Likelihood Method via the NR and the EM Algorithms", American Journal of Theoretical and Applied Statistics, 6(1): 1-9.

http://dx.doi.org/10.1155/2016/8246390.

http://interstat.statjournals.net/YEAR/2003/articles/0302004.pdf.

$\mathrm{Li}$, Yaowu and Li, Meihua, 2012. "Moment Estimation of the Parameters in Rayleigh Distribution with

Two Parameters", Communications in statistics theory and methods, 41(15): 2643-2660.

Mahdi, Smail and Cenac, Myrtene, 2006. "Estimating and Assessing the Parameters of the Logistic and Rayleigh Distributions from Three Methods of Estimation",Caribb. J. Math. Comput. Sci., 13: 25-34.

Mkolesia, A.C., C.R. Kikawa and M.Y. Shatalov, 2016. " Estimation of the Rayleigh Distribution Parameter",Transylvanian Review: XXIV(8): 1158-1163.

Rasheed, Huda A. and ArefRaghdaKh., 2017. "Bayes Estimator for Inverse Rayleigh Distribution under Generalized Weighted Loss Function",Mathematics and Statistics Journal, 3(1): 18 -24.

Rashid, M. Zahid and Akhter, A. Saeed, 2011. "Estimation Accuracy of Exponential Distribution Parameters", Pakistan Journal of Statistics and Operation Research, VII(2): 217-232.

Seo, Jung-In,Jeon, Jae-Woo and Kang, Suk-Bok, 2016. "Exact Interval Inference for the Two-Parameter Rayleigh Distribution Based on the Upper Record Values", Journal of Probability and Statistics, pp. (15).Article ID 8246390.Hindawi Publishing Corporation.

Taha, Taha Anwar, 2015. "On Reliability Estimation for the Rayleigh Distribution Based on Monte Carlo Simulation", International Journal of Science and Research (IJSR), 6(8): 1163-1167. DOI: 10.21275/ART20176044.

Walck, Christian, 2007. "Hand-book on Statistical Distributions for experimentalists",Internal Report SUFPFY/96-01,University of Stockholm.

Yahya Mahdi Al Mayali and rtifaaAbulKadhumAl_Shaibani, 2013. "A Comparison for Some of the Estimators of Rayleigh Distribution with Simulation", Journal of Karbala University, 11(4): 104-112. 